\title{
BOUNDED CURVATURE CLOSURE OF THE SET OF COMPACT RIEMANNIAN MANIFOLDS
}

\author{
I. G. NIKOLAEV
}

\section{INTRODUCTION}

In this note we consider the set of metric spaces which are the limits with respect to Lipschitz distance $d_{L}$ of compact connected $C^{\infty}$-Riemannian manifolds of curvature uniformly bounded above and below. We call this set "bounded curvature closure" (BCC).

It is well known that the limit spaces need not be $C^{2}$-Riemannian manifolds [P, Example 1.8]. Hence, the problem arising is to give a geometrical description of the BCC.

We solve this problem with the help of the theory of metric spaces of bounded curvature which A. D. Aleksandrov introduced more than 30 years ago [A] to construct the synthetic generalization of Riemannian geometry. Our principal result (Closure Theorem) states that the BCC consists of all compact Aleksandrov's spaces of bounded curvature.

A consideration of the $\mathrm{BCC}$ is definitely of independent interest, but due to Gromov's compactness theorem it has proved to be useful to consider the BCC in connection with different problems of Riemannian geometry. Now we are going to formulate the compactness theorem and explain the ways of its applications. First let us give necessary definitions.

Let $\left(\mathscr{M}_{1}, \rho_{1}\right),\left(\mathscr{M}_{2}, \rho_{2}\right)$ be metric spaces with metrics $\rho_{1}$ and $\rho_{2}, f:\left(\mathscr{M}_{1}, \rho_{1}\right) \rightarrow\left(\mathscr{M}_{2}, \rho_{2}\right)$ a Lipschitz map. Then

$$
\operatorname{dil} f=\sup \left\{\rho_{2}(f(x), f(y)) / \rho_{1}(x, y) \mid x, y \in \mathscr{M}_{1}, x \neq y\right\}
$$

is called dilatation of $f$.

Suppose that $\left(\mathscr{M}_{1}, \rho_{1}\right),\left(\mathscr{M}_{2}, \rho_{2}\right)$ are compact. Then

$$
d_{L}\left(\mathscr{M}_{1}, \mathscr{M}_{2}\right)=\inf \left\{|\ln \operatorname{dil} f|+\left|\ln \operatorname{dil} f^{-1}\right| \mid f \text { bi-Lipsch.hom. }\right\}
$$

Received by the editors October 18, 1989 and, in revised form, May 29, 1990. 1980 Mathematics Subject Classification (1985 Revision). Primary 53C20; Secondary 53C45, 51K10, 51F99. 
is the Lipschitz distance between $\mathscr{M}_{1}$ and $\mathscr{M}_{2}$ provided that biLipschitz homeomorphisms exist. Otherwise $d_{L}\left(\mathscr{M}_{1}, \mathscr{M}_{2}\right)=+\infty$ (see $[\mathrm{G}]$ ).

M. Gromov considered the class $M(n, d, V, \Lambda)$ of $n$-dimensional compact connected $C^{\infty}$-Riemannian manifolds $\mathscr{M}$ with diameter $\operatorname{diam}(\mathscr{M}) \leq d$, volume $\operatorname{Vol}(\mathscr{M}) \geq V>0$ and sectional curvatures $\left|K_{\mathscr{M}}\right| \leq \Lambda$. Gromov's compactness theorem states (see Theorems 8.25 and 8.27 of [G]):

Given a sequence $\left\{\mathscr{M}_{k}, k=1,2, \ldots\right\}$ in $M(n, d, V, \Lambda)$, there exists a subsequence $\left\{\mathscr{M}_{k_{1}}, l=1,2, \ldots\right\}$ and a $C^{0}$-Riemannian manifold $\mathscr{M}_{\infty}$ such that $\left\{\mathscr{M}_{k_{l}}\right\}$ converges to $\mathscr{M}_{\infty}$ in Lipschitz distance $d_{L}$; that is, $M(n, d, V, \Lambda)$ is precompact in a larger class of nonregular Riemannian manifolds.

As a typical and important example of an application of Gromov's theorem, let us consider the following result due to Berger:

Berger's theorem on almost 1/4-pinched manifolds [B2]. Let $\mathscr{M}$ be a compact simply connected $C^{\infty}$-Riemannian manifold of even dimension $n$. There exists a universal constant $E_{n}$ depending only on $n$ such that if $1 / 4-E_{n} \leq K_{\mathscr{M}} \leq 1$, then $\mathscr{M}$ is either homeomorphic to a standard sphere $S^{n}$ or diffeomorphic to a symmetric space of rank 1 .

Berger's theorem is proved by contradiction. He assumes to have a sequence of $C^{\infty}$-Riemannian manifolds $\left\{\mathscr{M}_{k}, k=1\right.$, $2, \ldots\}$ with pinching constants converging to $1 / 4$ which are not homeomorphic to $S^{n}$ or diffeomorphic to a symmetric space of rank 1. The compactness theorem is used to yield a " $1 / 4$-pinched" limit space $\mathscr{M}_{\infty}$ which is diffeomorphic to $\mathscr{M}_{k}$ for large $k$ due to Cheeger's finiteness theorem [Ch]. The main difficulty is to show that the limit space inherits the assertion of a well-known rigidity theorem [B1]. Hence, $\mathscr{M}_{\infty}$ is either homeomorphic to $S^{n}$ or diffeomorphic to a symmetric space of rank 1, a contradiction.

Durumeric [D] made use of the compactness theorem to generalize Berger's pinching theorems. For other applications of Gromov's theorem, see $[\mathrm{Br}]$ ("diameter-pinching") and [K] ("volumepinching").

We have seen that properties of a limit metric in the compactness theorem are crucial for applications. Due to the trivial part of the Closure Theorem (see also [P, P1]), a far-advanced theory of Aleksandrov's spaces of bounded curvature (for example, see survey $[\mathrm{ABN}]$ ) is applicable to limit spaces in Gromov's compactness 
theorem. In particular, $C^{1, \alpha}$-result by Peters $[\mathrm{P}]$ and Greene and $\mathrm{Wu}[\mathrm{GW}]$ follows from the earlier general Smoothness Theorem $(\S 3)$.

In the meantime, the Closure Theorem describes spaces of bounded curvature from the sequential point of view. As a consequence one can carry theorems of Riemannian geometry over metric spaces. Examples are given at the end of $\S 5$.

2. UPPER AND LOWER CURVATURES OF METRIC SPACE

For metric spaces, the concepts of (shortest) geodesic, (upper) angle between geodesics, and triangle made up of the geodesics are defined (see $[\mathrm{A}, \mathrm{R}]$ ).

The area $s(T)$ of a triangle $T$ is defined to be equal to the area of a Euclidean triangle of the same edge lengths. The excess $\delta(T)$ of a triangle $T$ is understood to be the sum of the angles at the vertices of $T$ minus $\pi$.

Define the upper and lower curvatures $\bar{K}(T)$ and $\underline{K}(T)$ of a triangle $T$ as follows. If $s(T) \neq 0$, then

$$
\bar{K}(T)=\underline{K}(T)=\delta(T) / s(T) .
$$

For a degenerate triangle (i.e. $S(T)=0$ ), set

$$
\begin{aligned}
& \bar{K}(T)= \begin{cases}+\infty & \text { if } \delta(T)>0 \\
-\infty & \text { if } \delta(T) \leq 0,\end{cases} \\
& \underline{K}(T)= \begin{cases}+\infty & \text { if } \delta(T) \geq 0 \\
-\infty & \text { if } \delta(T)<0 .\end{cases}
\end{aligned}
$$

The upper and lower curvatures of a locally compact metric space $(\mathscr{M}, \rho)$ with intrinsic metric $\rho$ at a point $P$ in $\mathscr{M}$ are introduced as follows:

$$
\bar{K}_{\mathscr{M}}(P)=\varlimsup \overline{\lim } \bar{K}(T), \underline{K}_{\mathscr{M}}(P)=\underline{\lim } \underline{K}(T), T \rightarrow P,
$$

where triangles $T$ contract arbitrarily to the point $P$.

The upper and lower curvatures of $(\mathscr{M}, \rho)$ are defined as

$$
\bar{K}(\mathscr{M})=\sup \left\{\bar{K}_{\mathscr{M}}(P)\right\}, \underline{K}(\mathscr{M})=\inf \left\{\underline{K}_{\mathscr{M}}(P)\right\}, P \in \mathscr{M} \text {. }
$$

\section{SPACES OF BOUNDED CURVATURE}

A locally compact metric space $(\mathscr{M}, \rho)$ with intrinsic metric is called a space of bounded curvature if it satisfies the conditions:

(i) The condition of local extendability of geodesic: For each point of $\mathscr{M}$ there is a ball of sufficiently small radius with 
center at this point such that if two points lying inside the ball can be joined by a geodesic, then this can be extended so that these points become interior points of the extended geodesic.

(ii) For each point $P \in \mathscr{M}$ the upper and lower curvatures at $P$ satisfy the inequalities: $\bar{K}_{\mathscr{M}}(P)<+\infty, \underline{K}_{\mathscr{M}}(P)>-\infty$.

V. N. Berestovskii proved that in spaces of bounded curvature it is possible to introduce a $C^{0}$-Riemannian structure (see [ABN]). The final statement about differential properties of a metric of a space of bounded curvature is:

Smoothness Theorem [N1, N2]. In a space of bounded curvature $(\mathscr{M}, \rho)$ it is possible to introduce the structure of a Riemannian manifold with the help of local harmonic coordinates, which form an atlas $\mathrm{H}_{0}$ of smoothness $C^{3, \alpha}$, and the metric tensor in the harmonic coordinates belongs at least to $W_{q}^{2} \cap C^{1, \alpha}$ for each $q \in$ $[1,+\infty), \alpha \in(0,1)$.

Here by $W_{q}^{2}$ we denote Sobolev's class of functions having second generalized derivatives summable to the power $q$.

For further details, see survey [ABN].

Remark. Plaut generalized Berestovskii theorem in [P1] where he stated that a finite-dimensional metric space of bounded curvature without the condition (i) is a manifold with boundary. A smoothness theorem for metric spaces of bounded curvature with boundary is not known.

\section{APPROXIMATION THEOREM}

Let $\langle\mathscr{M}, g\rangle$ be a connected Riemannian manifold $\mathscr{M}$ with metric tensor $g$. By $\rho(g)$ we denote the intrinsic metric on $\mathscr{M}$ constructed by means of $g$. For a $C^{\infty}$-atlas containing in $H_{o}$ (which exists due to [W]) we keep the notation $H$.

Approximation Theorem. Let $\left(\mathscr{M}, \rho\left(g_{o}\right)\right)$ be a space of bounded curvature. Then there exists a sequence of $C^{\infty}$-Riemannian metrics $\left\{g_{m} \mid m=1,2, \ldots\right\}$ on the $C^{\infty}$-manifold $(\mathscr{M}, H)$, for which the following properties are realized:

(i) The maps $i_{m}:\left(\mathscr{M}, \rho\left(g_{m}\right)\right) \rightarrow\left(\mathscr{M}, \rho\left(g_{o}\right)\right)$,

$$
i_{m}(P)=P, P \in \mathscr{M} ; m=0,1,2, \ldots
$$

are bi-Lipschitz homeomorphisms for which

$$
\lim _{m \rightarrow \infty} \operatorname{dil} i_{m}=\lim _{m \rightarrow \infty} \operatorname{dil} i_{m}^{-1}=1,
$$


(ii) $\varlimsup \bar{K}_{m} \leq \bar{K}_{o}, \underline{\lim } \underline{K}_{m} \geq \underline{K}_{o}, m \rightarrow \infty$, where by $\bar{K}_{m}$ and $\underline{K}_{m}$ we denote the upper and lower curvatures of spaces $\left(\mathscr{M}, \rho\left(g_{m}\right)\right), m=0,1,2, \ldots$.

The approximation sequence is constructed with the help of Rham's smoothness operator [Rh].

The Smoothness Theorem enables us to define the sectional curvatures $K_{f}(u \wedge v)$ of $\left\langle\mathscr{M}, g_{o}\right\rangle$ which are formally calculated "almost everywhere" on $\mathscr{M}$ by $g_{o}$. We introduce the formal upper curvature as

$$
\bar{K}_{f}(\mathscr{M})=\inf _{\mathscr{O}} \sup _{P \in \mathscr{M} \backslash \mathscr{O}}\left\{\sup _{\substack{u, v \in \mathscr{M}_{p} \\ u \wedge v \neq 0}}\left\{K_{f}(u \wedge v)\right\}\right\}
$$

where inf is taken over all sets $\mathscr{O} \subset \mathscr{M}$ of null $n$-dimensional Hausdorff measure $(n=\operatorname{dim} \mathscr{M})$.

In a similar way, it is possible to define the formal lower curvature $\underline{K}_{f}(\mathscr{M})$.

One can prove (ii) for $\bar{K}_{f}(\mathscr{M})$ and $\underline{K}_{f}(\mathscr{M})$ making use of the Smoothness Theorem and a technique of the theory of functions with generalized derivatives. Thus, the last problem is to prove that

$$
\bar{K}_{f}(\mathscr{M}) \leq \bar{K}(\mathscr{M}), \underline{K}_{f}(\mathscr{M}) \geq \underline{K}(\mathscr{M}) .
$$

These inequalities can be proved with the help of a generalization of second variation formula [N3] to spaces of bounded curvature.

\section{Closure Theorem}

A compact metric space $\left(\mathscr{M}_{o}, \rho_{o}\right)$ is said to be a bounded curvature limit of the sequence of compact metric spaces $\left\{\left(\mathscr{M}_{m}, \rho_{m}\right) \mid m\right.$ $=1,2, \ldots\}$ (notation $\left(\mathscr{M}_{o}, \rho_{o}\right)=($ b.c. $\left.) \lim \left(\mathscr{M}_{m}, \rho_{m}\right)\right)$ if:

(i) The spaces $\left(\mathscr{M}_{m}, \rho_{m}\right)$ converge to the space $\left(\mathscr{M}_{o}, \rho_{o}\right)$ in Lipschitz distance $d_{L}$.

(ii) For some natural number $m_{o}$ the following inequalities hold: $-\infty<\inf \left\{\underline{K}\left(\mathscr{M}_{m}\right)\right\} \leq \sup \left\{\bar{K}\left(\mathscr{M}_{m}\right)\right\}<+\infty, m \geq$ $m_{o}$.

Denote by $M_{c}$ the set of all compact metric spaces with intrinsic metric. The notion of (b.c.)lim just defined induces in $M_{c}$ a sequential topology $t_{\text {b.c. }}$ which turns out to be a Fréchet topology [E]. In particular a closure of a set $U \subseteq M_{c}$ in the topological space 
$\left(M_{c}, t_{\text {b.c. }}\right)$ consists of all (b.c.) limits of convergent sequences of $U$.

Let $M_{R}$ be the set of all connected compact $C^{\infty}$-Riemannian manifolds and $M_{A}$ be the set of compact Aleksandrov's spaces of bounded curvature.

Closure Theorem. The closure of $M_{R}$ in the topological space $\left(M_{c}, t_{\text {b.c. }}\right)$ (i.e., the BCC) coincides with $M_{A}: \bar{M}_{R}=M_{A}$.

The nontrivial part of the Closure Theorem $\left(M_{A} \subseteq \bar{M}_{R}\right)$ is a direct consequence of the Approximation Theorem. To prove the inclusion $M_{A} \supseteq \bar{M}_{R}$ one has to note that the volumes of each (b.c.) convergent sequence of Riemannian manifolds uniformly differ from 0 (thus, the same is valid for the injectivity radii). Hence, the inclusion $M_{A} \supseteq \bar{M}_{R}$ is trivial.

Corollary 1. (Addendum to Gromov's compactness theorem.) The set $\bar{M}(n, d, V, \Lambda)$ of compact spaces of bounded curvature with the same restrictions upon dimension, diameter, volume, and curvature as in the compactness theorem is compact with respect to Lipschitz distance $d_{L}$.

Corollary 2. (Metric Version of the Sphere Theorem.) Let $(\mathscr{M}, \rho)$ be a finitely-compact simply-connected metric space with intrinsic metric satisfying the condition of local extendability of geodesic. If

$$
1 / 4<\underline{K}(\mathscr{M}) \leq \bar{K}(\mathscr{M}) \leq 1,
$$

then $\mathscr{M}$ is a manifold homeomorphic to sphere $S^{n}$ for some $n \geq 2$.

\section{ACKNOWLEDGMENT}

The author wishes to thank Ju. D. Burago for fruitful and stimulating discussions.

\section{REFERENCES}

[A] A. D. Aleksandrov, Uber eine Verallgemeinerung der Riemannschen Geometrie, Schr. Forschungsinst. Math. 1 (1957), 33-84.

[ABN] A. D. Aleksandrov, V. N. Berestovskii, and I. G. Nikolaev, Generalized Riemannian spaces, Russian Math. Surveys 41(3) (1986), 1-54.

[B1] M. Berger, Les variétés Riemanniennes 1/4-pincées, Ann. Scuola Norm. Sup. Pisa Cl. Sci. 14 (1960), 161-170.

[B2] _- Sur les variétés Riemanniennes pincées juste audessous de 1/4, Ann. Inst. Fourier (Grenoble) 33(2) (1983), 135-150.

[Br] D. Brittain, Lecture at Luminy, France, July 1984. 
[Ch] J. Cheeger, Finiteness theorems for Riemannian manifolds, Amer. J. Math. 92 (1970), 61-74.

[D] O. Durumeric, A generalization of Berger's theorem on almost 1/4-pinched manifolds. II, J. Differential Geom. 26(1) (1987), 101-139.

[E] R. Engelking, General topology, Państwowe Wydawnictwo Naukowe, Warszawa, 1977 (Monogr. matematyczne; t. 60).

[GW] R. E. Greeene and H. Wu, Lipschitz convergence of Riemannian manifolds, Pacific J. Math. 131(1) (1988), 119-141.

[G] M. Gromov, J. Lafontaine, and P. Pansu, Structures métriques pour les variétés Riemanniennes, Cedic/Fernand Nathan, Paris, 1981.

[K] A. Katsuda, Gromov's convergence theorem and its application, Nagoya Math. J. 100 (1985), 11-48.

[N1] I. G. Nikolaev, Parallel translation and smoothness of the metric of spaces of bounded curvature, Soviet Math. Dokl. 21 (1980), 263-265.

[N2] _ Smoothness of the metric of spaces with bilaterally bounded curvature in the sense of A. D. Aleksandrov, Siberian Math. J. 24 (1983), 247-263.

[N3] _ The Synge's formula for geodesic variations in a space of bounded curvature by A. D. Aleksandrov, preprint (Russian).

[P] S. Peters, Convergence of Riemannian manifolds, Compositio Math. 62(1) (1987), 3-16.

[P1] C. Plaut, A metric characterization of manifolds with boundary, preprint.

[Rh] G. de Rham, Variétés différentiables: Formes, courants, formes harmoniques, Hermann et Cie, Paris, 1955.

[R] H. Rinow, Die innere Geometrie der metrischen Raume, Springer-Verlag, Berlin-Heidelberg-New York, 1961.

[W] H. Whitney, Differential manifolds, Ann. of Math. 37(3) (1936), 645-680.

Institute of Mathematics, Siberian Branch of the ACADEMy of Sciences OF THE USSR, UNIVERSITETSKII PROSP. 4, 630090 NOVOSIBIRSK 90 USSR 
